\title{
Germanica
}

\section{Paul Verlaine und die Rezeption der französischen Moderne in der DDR}

Verlaine et la réception de la modernité française en RDA

Verlaine and the East German Reception of French Modern Literature

\section{Tilman Schreiber}

\section{OpenEdition}

Journals

Édition électronique

URL : http://journals.openedition.org/germanica/3490

DOI : 10.4000/germanica.3490

ISSN : 2107-0784

Éditeur

Université de Lille

Édition imprimée

Date de publication : 30 décembre 2016

Pagination : 135-142

ISBN : 9782913857384

ISSN : 0984-2632

\section{Référence électronique}

Tilman Schreiber, „Paul Verlaine und die Rezeption der französischen Moderne in der DDR“, Germanica

[Online], 59 | 2016, Online erschienen am: 30 Dezember 2018, abgerufen am 06 Januar 2021. URL:

http://journals.openedition.org/germanica/3490 ; DOI: https://doi.org/10.4000/germanica.3490

\section{(c) Tous droits réservés}




\title{
Paul Verlaine und die Rezeption der französischen Moderne in der DDR
}

\author{
Tilman SCHREIBER \\ Friedrich-Schiller-Universität Jena
}

\section{Methodologische Vorbemerkungen}

Die Frage nach der Rezeption der französischen Moderne in der DDR ist im besonderen Maße eine Frage nach politisch-kultureller Identität. Sie berührt zum einen das Selbstverständnis des Staates im Allgemeinen, also seine sich in Opposition zur bundesrepublikanischen ,Geschichte verstehenden Bestrebungen der Traditionsstiftung. Zum anderen betrifft sie natürlich das Selbstverständnis vieler ,Kulturschaffender', d.h. von Autoren, Verlegern, Publizisten und allen sonstigen Lesern. Etwas allgemeiner gesprochen bezeichnet der Begriff der ,Rezeption' also ein ganzes System von Prozessen, die sich sowohl zwischen unterschiedlichen Instanzen als auch auf jeweils unterschiedliche Weise vollziehen. Damit besitzt er streng genommen den Charakter eines Kollektivsingulars.

An diese erste Überlegung ist eine zweite anzuschließen, die sich nicht so sehr auf den allgemeinen Vorgang der Aneignung als vielmehr auf die Texte bezieht, die unter dem Signum der, französischen Moderne' firmieren. Diese sind - zumindest tendenziell - in zwei Gruppen zu unterteilen. Der ersten Gruppe gehören diejenigen Autoren an, die bereits vor Entstehung der DDR klassisch geworden waren. So sind beispielsweise Baudelaire und seine Ästhetik in den 1950er 
Jahren des 20. Jahrhunderts bereits so stark in den (teilweise bis in die Weimarer Republik zurückreichenden) Bildungsgeschichten der DDR-Autoren und -Leser verankert, dass sich die Frage nach der grundsätzlichen Kenntnis bzw. Möglichkeit der formsprachlichen Adaption nicht mehr ernsthaft stellt. Die Formensprache der frühen Moderne war vielmehr von Beginn an als ästhetisches Potential im ostdeutschen Staat vorhanden. Diese potentielle Verfügbarkeit ist im Falle der zweiten Autoren-Gruppe nicht mehr selbstverständlich. Jener gehören diejenigen in der Regel zeitgenössischen Schriftsteller wie z.B. Nathalie Sarraute an, deren Kanonisierungsprozess noch nicht abgeschlossen war.

Diese vergleichsweise allgemeinen Überlegungen machen somit bereits deutlich, dass die Frage nach der ostdeutschen Rezeption der französischen Moderne in dieser grundsätzlichen Form nicht zu beantworten ist. Vielmehr ist nach den Rezeptionen bzw. den Rezeptionsinstanzen und ihrer diskursiven Prägekraft zu fragen.

Dieser methodologische Ansatz soll im Folgenden am Beispiel Paul Verlaines in die rezeptionsanalytische Praxis überführt werden. Im Zuge dessen findet eine Charakterisierung der den Interpretationen von Verlaines Biographie und seinem Euvre zugrunde liegenden Argumentationsstrategien statt, wie sie in den Vor- und Nachworten der verschiedenen Ausgaben zum Einsatz kommen. Dabei liegt der inhaltliche Fokus zwar selbstredend auf denjenigen Editionen des Franzosen, die in der DDR erschienen sind, den eigentlichen Ausgangspunkt der Argumentation bildet jedoch die von Stefan Zweig besorgte Werkausgabe von 1922. Dieses Vorgehen begründet sich dadurch, dass jener Edition im Hinblick auf die Rezeptionsgeschichte Verlaines vor 1949 ein exemplarischer Charakter zukommt, sich auf ihrer Grundlage also der Maßstab bestimmen lässt, an dem sich die ostdeutschen Ausgaben auf die eine oder andere Weise orientiert haben.

\section{Stefan Zweig - Verlaine als, deutscher' Lyriker}

Die 1922 im Insel Verlag erschienene zweibändige Werkausgabe zeugt - bereits 26 Jahre nach dem Tod des Dichters - von einer intensiven Rezeption Verlaines in Deutschland. So finden sich im Inhaltsverzeichnis des Bandes zur Poesie eine große Anzahl unterschiedlicher zum Teil prominenter Übersetzer. Neben Arbeiten von Zweig selbst werden u.a. Nachdichtungen von Hermann Hesse, Stefan George und Rainer Maria Rilke aufgelistet. Dieser, alleine auf Grundlage des Verzeichnisses der Übersetzer gewonnene Eindruck, erhärtet sich bei der Lektüre des ca. zwanzigseitigen Nachwortes. Dort entwickelt Zweig die Idee von Verlaine als einem dezidiert, deutschen' Lyriker, der mit seinen Werken 
in der Tradition des romantischen Liedes steht ${ }^{1}$. Damit führt er einen Topos in die Rezeption dieses Dichters ein, auf den in den folgenden Ausgaben immer wieder Bezug genommen wird.

Die beiden anderen Auffassungen, die im Zentrum des Zweigschen Nachwortes stehen, sind die der charakterlichen Fragwürdigkeit Verlaines und der poetischen Mittelmäßigkeit vieler seiner Texte. Erstere wird dabei vor allem im Kontrast zu Rimbaud - eine Verbindung, die in der Folge mit wechselnder Nachdrücklichkeit gezogen werden wird - konstatiert: „Nicht wie Rimbaud, sein Verführer und Begleiter in den Strotterjahren[sic], atmet er, ein echter Prinz Vogelfrei, nur wohl auf fremder Streu, in fremder Luft: Verlaine bleibt zeitlebens Bohémien wider Willen 2". An anderen Stellen finden sich noch weitaus deutlichere Formulierungen, wenn Zweig Verlaine als ,Schwächling' bezeichnet oder bezogen auf einzelne Werke ausruft: „Ach, was für schlechte

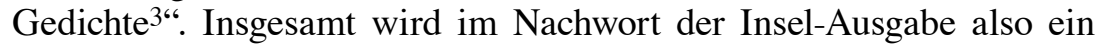
sehr gemischtes Urteil über den Dichter gefällt. Zweig zufolge ist Verlaine kein literarischer Heros, sondern vielmehr eine Figur, die ihre literaturgeschichtliche Relevanz aus dem in einigen poetisch hochstehenden Gedichten ausgedrückten Eingeständnis der eigenen Schwäche gewinnt.

Auf einer allgemeineren Ebene zeugt die zweibändige Werkausgabe von 1922 also für eben jenen Kanonisierungsgrad der Autoren der frühen Moderne, der bereits in den einleitenden Überlegungen skizziert wurde. Die Insel-Edition bildet - das bestätigt das Verzeichnis der Nachdichter sowie der Herausgeber Zweig mit seinem Selbstverständnis als literaturvermittelnde Instanz - bereits eine Art vorläufigen Endpunkt in der deutschen Verlaine-Rezeption. Das Nachwort präsentiert den französischen Dichter, ungeachtet der qualitativen Heterogenität seiner lyrischen Produktion, als festen kulturellen Bestandteil der zeitgenössischen deutschen Identität. Umso interessanter ist also die Frage, wie in der DDR, insbesondere vor dem Hintergrund des Versuches, einen , anti-bürgerlichen' Literaturkanon zu etablieren, mit diesem ästhetischen Erbe umgegangen wurde.

\section{Stephan Hermlin - Der, spätbürgerliche`Verlaine}

Auf den ersten Blick mag es überraschen, dass ausgerechnet Verlaine anstelle von Baudelaire oder Rimbaud als erster Dichter der frühen

1. - Vgl. Paul Verlaine, Gesammelte Werke. Bd. 1. Gesammelte Gedichte. Eine Auswahl der besten Übertragungen, hrsg. von Stefan Zweig, Leipzig, Insel Verlag, 1922, S. 9.

2. - Paul Verlaine, Gesammelte Werke. Bd.2. Lebensdokumente, hrsg. von Stefan Zweig, Leipzig, Insel Verlag, 1922, S. 5.

3. - Ebd., S. 19. 
Moderne in der DDR erscheint. Dieser Eindruck wird jedoch ziemlich schnell relativiert, wenn man einen Blick in das Nachwort der 1970 von Stephan Hermlin bei Reclam besorgten Ausgabe wirft. Dort heißt es zu Beginn: „In Deutschland ist von den großen französischen Lyrikern des 19. Jahrhunderts Verlaine am besten bekannt, am sichersten über-

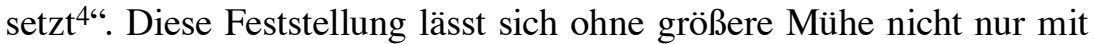
den Ausführungen Zweigs in der Insel-Ausgabe von 1922 in Verbindung bringen, sondern ebenso mit der prominenten Rolle, die Verlaine in der Erstausgabe der Anthologie zur Französischen Lyrik von Baudelaire bis zur Gegenwart (1961) einnimmt ${ }^{5}$. Scheinbar - und der Rest des Nachwortes bestätigt diesen Eindruck - wird in der Ausgabe von 1970 ziemlich konsequent die Rezeptionstradition von vor 1949 fortgeführt. Bemerkenswert ist dies nicht so sehr hinsichtlich des Herausgebers und seines Selbstverständnisses als ,spätbürgerlicher Schriftsteller ${ }^{6}$, sondern im Hinblick auf die Tatsache, dass anscheinend auch im Rahmen des Druckgenehmigungsverfahrens kein Anstoß an der Fortführung dieser Kanonisierungs- und Deutungstradition genommen wurde.

Dieser Eindruck erhärtet sich, wenn man die entsprechenden Druckgenehmigungsakten aus dem Bestand des Bundesarchivs konsultiert. So besteht seitens des HV-Gutachters weder gegen die Auflagenhöhe (immerhin 10000 Exemplare) noch gegen das Nachwort ein Einwand. Verlag und Herausgeber werden lediglich ersucht, in der Zusammenstellung der Texte die religiöse Komponente in Verlaines Dichtung etwas zurückzunehmen, was schließlich dazu führt, dass Mon Dieu m'a dit gegen ein Gedicht ausgetauscht wird, das im engeren Bezug zur Commune steht ${ }^{7}$. In Verbindung damit wird jedoch nicht gefordert, dass Hermlin auch in seinem Nachwort stärker auf dieses politische Ereignis eingeht, beispielsweise dessen ,besondere Relevanz für

4. - Paul Verlaine, Gedichte, hrsg. von Stephan Hermlin, Leipzig, Reclam, 1970, S. 93.

5. - Wie der Titel bereits andeutet, versteht sich diese Ausgabe als Versuch, die ästhetische und weltanschauliche Provenienz der zeitgenössischen Lyrik aus der französischen Dichtung um 1900 zu rekonstruieren. Bemerkenswert an dieser Anthologie ist der Umstand, dass sie durch die Auswahl der Gedichte und den Paratext auf ein insbesondere durch Hugo Friedrichs Die Struktur der modernen Lyrik (1956) entwickeltes, d.h. westdeutsches literarturhistorisches Narrativ zurückgreift, das nicht nur in späteren Publikationen fortgeführt wird, sondern seine Wirkungsmacht bis in die gegenwärtige Forschung (nicht nur) zur DDR-Literatur behält. So ist die Frage nach der ,französischen Moderne in der DDR` streng genommen immer noch jener Interpretation verpflichtet, die unter den Vorzeichen des historischen Materialismus und der Leitidee des Humanismus eine notwendige literarische Entwicklung proklamiert.

6. - Art. Hermlin, Stephan, In: Metzler-Lexikon DDR-Literatur. Autoren Institutionen - Debatten, hrsg. von Michael Opitz u. Michael Hofmann, Stuttgart u. Weimar, J.B. Metzler, 2009, S. 126r.

7. - BArch DR 1/340/143/70, pag. 1. 
Leben und Dichtung' Verlaines herausstellt. Konsequenterweise heißt es dort lapidar: „Die Commune hat übrigens im Werk beider Dichter [i.e. Verlaine und Rimbaud, TS] Spuren hinterlassen ${ }^{8 “}$. Insgesamt der Eindruck wird durch die Druckgenehmigungsakten noch einmal unterstrichen - sind die Bemühungen, Verlaine ideologisch auf Linie zu bringen, also sehr überschaubar. Die für sein Euvre typische lyrische Diversität sowie der nach DDR-Einschätzung eigentlich ,formalistische Zug mancher Gedichte wurden offensichtlich nicht als problematisch empfunden.

Als weiterer Beleg für diese grundsätzliche Akzeptanz kann das Nebeneinanderexistieren von ideologisch sehr unterschiedlich orientierten Interpretationen des Verlainschen Euvres im öffentlichen Diskurs angeführt werden. So ist es gar innerhalb eines Verlages (in diesem Fall des in der DDR äußerst populären Reclam-Verlages) möglich, sowohl eine tendenziell marxistische als auch eine klassisch-literaturwissenschaftliche - also eigentlich ,bürgerliche ${ }^{6}$ - Deutung zu publizieren. Erstere wird durch Kurt Schnelle in der Anthologie zur Französischen Lyrik von 1961 gegeben, während letztere durch Hermlins Nachwort der Gedichte-Ausgabe von 1970 vorgenommen wird. Dass diese Parallelität der unterschiedlichen, aber an sich gleichwertigen Rezeptionsinstanzen nicht nur aus einer ungewöhnlichen historischen Konstellation heraus resultiert, wird bereits dadurch deutlich, dass beide Bände in den folgenden Jahren immer wieder neu aufgelegt wurden. So verändert sich zwar die Zusammenstellung der Anthologie zur Französischen Lyrik zu einem nicht unbedeutenden Ausmaß, die Gewichtung der Dichter sowie die Interpretation Verlaines im Nachwort bleiben jedoch grundsätzlich gleich. Ähnliches gilt für die von Hermlin besorgte Ausgabe der Gedichte, die 1980 in einer zweiten, unveränderten Auflage erscheint.

\section{Manfred Starke - Der, versachlichte' Verlaine}

Die genannten Editionen stellen jedoch nicht die beiden einzigen Deutungsangebote dar, die im öffentlichen Diskurs dieser Jahre zu finden sind. Jener wurde 1977 durch eine weitere, von Manfred Starke herausgegebene Verlaine-Ausgabe bereichert, die als erste explizit mit dem Anspruch auftrat, das Bild des Dichters - angefangen bei einer Neuübersetzung durch Sigmar Löffler - zu ,versachlichen ${ }^{9}$.

8. - Verlaine, Gedichte, 1970, a.a.O., S. 95.

9. - So der genaue Wortlaut aus der der Druckgenehmigungsakte entstammenden Stellungnahme des Verlages. Vgl. BArch DR $1 / 260 / 11 / 77$, pag. 2. Mit Löffler wird dabei auf einen Übersetzer zurückgegriffen, der sich in der DDR bereits mit einer Neuübertragung von Baudelaires Fleurs du mal (1973) einen Namen gemacht hatte.

Es ist eventuell sogar dieser Impetus - und nicht die hohe Nachfrage -, der dazu führt, dass diese Ausgabe in einer Auflage von 20000 Exemplaren erscheint. Diese ist 
In diesem Zusammenhang ist eine vergleichende Betrachtung der Nachworte dieser und der zuvor besprochenen Ausgaben erhellend. So rückt Starke sehr viel entschiedener den ,sozialistischen Verlaine“ in den Fokus der Betrachtung, wobei er insbesondere auf dessen Beschäftigung mit politisch links stehenden Theoretikern und seine Parteinahme für die Commune eingeht. Gerade im Bezug auf letztere wird der Unterschied zwischen dieser Edition und den vorhergegangenen deutlich. So liest sich Starkes Aussage „Verlaine hatte selbst an der revolutionären Arbeit der Commune teilgenommen ${ }^{10 " ~ d o c h ~ e n t s c h i e d e n ~ a n d e r s ~ a l s ~ H e r m l i n s ~}$ Einschätzung: „Die Commune hat übrigens im Werk beider Dichter [i.e. Verlaine und Rimbaud, TS] Spuren hinterlassen ${ }^{11 “ .}$

Diese punktuelle Umwertung steht dabei symptomatisch für den Versuch einer grundsätzlichen Neuinterpretation Verlaines. So werden von Starke im Hinblick auf Biographie und Euvre zwar bereits bekannte Charakteristika des Franzosen herausgearbeitet, diese jedoch dezidiert vor dem Hintergrund eines marxistischen Geschichtsbildes gedeutet. Ähnlich wie in den bereits analysierten Nachworten geht es ihm im Kern um Verlaines charakterliche wie weltanschauliche Unstetigkeit sowie die sehr unterschiedliche Qualität seines poetischen Schaffens. Das Neue bei Starke besteht nun darin, dass er Verlaines gesamte Existenz auf den ,ihm innewohnenden Widerspruch zwischen Bohème und Bourgeois ${ }^{12 "}$ "zu beziehen versucht. Relevanz gewinnt der Verfasser der Romances sans paroles vor dem Hintergrund dieser Einschätzung durch den sich in seiner Lyrik und seiner Biographie artikulierenden Krisenzustand der spätbürgerlichen Gesellschaft und den Umstand, dass er dieser zumindest teilweise in Biographie und Ästhetik entgegensteht ${ }^{13}$. In Starkes Lektüre bestätigt der Autor Verlaine also den Geschichtsverlauf, wie er durch den historischen Materialismus angenommen wird - er erlangt einen symptomatischen Erkenntniswert.

Bemerkenswerterweise schließt Starkes mit 50 Seiten sehr umfangreiches Nachwort jedoch keineswegs mit dieser Wertung. Vielmehr steht am Schluss, wo traditionsgemäß die Frage nach dem gestellt wird, was von einem Künstler bleibt, die eigentlich weltanschaulich neutrale Würdigung einer, ,rhythmisch und klanglich ungebundenen Dichtung ${ }^{14 “ “ . ~}$ Bezeichnend ist darüber hinaus - um die Ausgabe von 1977 selbst zu verlassen -, dass Verlaine auch für Starkes Anthologie Der Untergang

damit doppelt so hoch wie diejenige der Hermlin-Ausgabe von 1970. Vgl. ebd. pag. 1.

10. - Paul Verlaine, Poetische Werke, hrsg. von Manfred Starke, Leipzig, Insel Verlag, 1977, S. 568.

11. - Verlaine Gedichte 1970, a.a.O., S. 95.

12. - Verlaine Poetische Werke 1977, a.a.O., S. 569.

13. - Vgl. ebd. S. 558 u. 584.

14. - Ebd. 
der romantischen Sonne (erschienen 1984) eine vergleichsweise geringe Rolle spielt. Dieser erwähnt den Verfasser der Art poétique in seinem neunzigseitigen Vorwort, in dem es immerhin darum geht, ,das aktuelle Interesse dieser Tradition [i.e. der französischen Moderne, TS] für die sozialistische Dichtung zu klären ${ }^{15 “,}$, an keiner Stelle. Und auch als Autor ist der Franzose lediglich mit zwei kurzen Texten vertreten.

Einerseits ist Verlaine also ,nicht so ein radikaler Neuerer wie Rimbaud oder Mallarmé16" und für die zeitgenössische sozialistische Dichtung von sehr geringem Interesse. Andererseits wird jedoch die Notwendigkeit gesehen, der ,bürgerlichen' Interpretation dieses Autors eine ,versachlichte' gegenüberzustellen, die jedoch mit einer klassischliteraturwissenschaftlichen Würdigung schließt. Im Endeffekt bleibt also selbst Starkes Versuch einer, Versachlichung' des Verlaine-Bildes - wenn auch ungewollt - der Einschätzung verpflichtet, mit der Hermlin das Nachwort seiner Ausgabe einleitet. Auch in der Ausgabe von 1977 wird somit jene Rezeptionstradition fortgeführt, die bis wenigstens 1922 zurückreicht.

\section{Anfangs- und Endpunkt - Der ,bibliophile 'Verlaine}

1983 bringt der Insel Verlag die Neuauflage einer ehemals bibliophilen Verlaine-Ausgabe auf den Markt, die 1906 im eigenen Haus erstellt worden war. Diese versammelt ausgewählte Gedichte in der Übertragung Wolf von Kalckreuths, die durch Jugendstil-Illustrationen von Wilhelm Wulff ergänzt wurden. Weder die ursprüngliche Edition noch die Neuauflage ist mit einem Nachwort versehen. In der dazugehörigen Druckgenehmigungsakte heißt es diesbezüglich seitens des Verlages schlicht: „Da Verlaines Werk mehrfach in unserem Lande auch kommentiert erschienen ist, und der Band als Nachdruck gedacht ist, hat

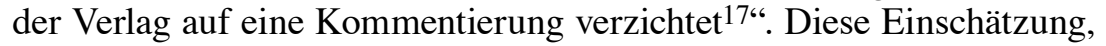
der äußerst geringe Gesamtumfang der Druckgenehmigungsakte sowie die generelle Problemlosigkeit, mit der der Veröffentlichungsprozess der Kalckreuth-Ausgabe ablief, fügen sich ziemlich genau in das Bild ein, das in dieser Skizze hinsichtlich der ostdeutschen Rezeption Verlaines entworfen wurde.

So war der Dichter schon vor 1949 gerade in Deutschland sehr stark kanonisiert und als Teil des eigenen kulturellen Erbes adaptiert worden. Ähnlich wie Baudelaire, Rimbaud und andere Autoren der frühen Moderne bildete Verlaine bereits einen festen Bestandteil der allge-

15. - Manfred Starke (Hrsg.), Der Untergang der romantischen Sonne. Ästhetische Texte von Baudelaire bis Mallarmé, Leipzig u. Weimar, Gustav Kiepenheuer, 1984, S. 7.

16. - So Starkes Urteil im Nachwort seiner Verlaine Ausgabe. Verlaine Poetische Werke 1977, a.a.O., S. 584.

17. - BArch DR $1 / 260 / 40 / 83$, pag. 3. 
meinen Bildungsgeschichte. Dementsprechend gab es in der DDR auch nie den Versuch, ihn trotz seiner weltanschaulichen Wankelmütigkeit und teilweisen literarischen Mediokrität aus dem Kanon wieder auszuschließen. Zwar existierten, wie anhand der Lyrik-Anthologie von 1961 und der Starke-Ausgabe von 1977 gezeigt wurde, durchaus Bestrebungen, ihn im Rahmen einer marxistischen Deutung zu adaptieren. Aber auch diese Versuche gingen letzten Endes lediglich als Deutungsvarianten in den öffentlichen Diskurs ein. Insgesamt gestaltet sich die Rezeption Verlaines in der DDR also als vergleichsweise konsequente Fortsetzung der Aneignungstradition von vor 1949. In dieser Linie bildet die InselAusgabe von 1983 einen weiteren Markstein. Mit ihr ist die nächste Stufe der Historisierung erreicht: Nicht nur der Autor und sein Werk bilden Gegenstände des historisch-ästhetischen Interesses, sondern ebenso seine Ausgaben.

Auf einer allgemeineren Ebene steht Verlaine gleichzeitig für eine spezifische Form der Rezeption der frühen französischen Moderne in der DDR. Um ihn wurden keine großen Debatten, keine heftigen ideologischen Kämpfe geführt. Im Kern der Beschäftigung mit diesem Autor stand wie in den Jahrzehnten zuvor das ,schöne' Gedicht - mit bzw. trotz all der positiven wie negativen Konnotationen, die diesem Begriff je nach Standpunkt anhaften. 